\title{
REPRESENTAÇÕES SOCIAIS DE MULHERES AMAZÔNIDAS SOBRE 0 EXAME PAPANICOLAU: IMPLICAÇÕES PARA A SAÚDE DA MULHER ${ }^{a}$
}

\author{
Social Representations of women amazônidas on examination \\ papanicolau: implications for the health of the woman \\ Representaciones Sociales de muj eres amazônidas sobre el \\ examen papanicolau: implicaciones para la salud de la mujer
}

\author{
Sílvio Éder Dias da Silva ${ }^{1}$ \\ Vera Lúcia de Azevedo Lima ${ }^{4}$
}

\author{
Esleane Vilela Vasconcelos ${ }^{2}$ \\ Francileni da Luz Carvalho ${ }^{5}$
}

\author{
Mary Elizabeth de Santana ${ }^{3}$ \\ Dayse Farias Mar $^{6}$
}

\section{RESUMO}

Esta pesquisa tem como objetivos descrever as representações sociais de mulheres amazônidas sobre o exame Papanicolau e analisar as implicações desta para o cuidado de si mesmas. Trata-se de um estudo qualitativo-exploratório com o uso da Teoria das representações sociais. A coleta de dados foi realizada no período de janeiro a dezembro de 2007, com o emprego de duas técnicas: a entrevista semi-estruturada com perguntas abertas e a observação livre. Para a interpretação desses dados foi utilizada a técnica de análise de conteúdo temático. A pesquisa teve como resultado três unidades temáticas, assim denominadas: 0 exame Papanicolau - um cuidado com a saúde da mulher; Tabus e crenças sobre o exame Papanicolau; e Uma prática de cuidado de si mesma: o exame Papanicolau. No estudo observamos que as mulheres temem muito ter câncer cérvico-uterino e, por esse motivo, representam o exame Papanicolau como uma prática de cuidado de si mesma.

Palavras-chave: Enfermagem. Neoplasias uterinas. Esfregaço vaginal. Saúde da mulher.

\begin{abstract}
This research has as objective to describe the social representations of women amazônidas on the Papanicolau examination and to analyze the implications of this for the same care of itself. One is about a qualitative-exploratório study with the use of the Theory of the social representations. The collection of data was carried through in the period from January through December of 2007, with the use of two techniques: the interview half-structuralized with open questions and the free comment. For the interpretation of these data the technique of analysis of thematic content was used. The research had as a result, three thematic units, thus called: The Papanicolau examination - a care with the health of the woman; Taboos and beliefs on the Papanicolau examination; and practical One of same care of itself: the Papanicolau examination. In the study we observe that the women are afraid of having uterine neoplasm for this reason, the Papanicolau examination represents a practical care of itself.
\end{abstract}

Keywords: Nursing. Uterine Neoplasm. Vaginal Smears. Health of the Woman.

\section{Resumen}

Esta investigación tiene como objetivos describir las representaciones sociales de mujeres amazônidas sobre el examen Papanicolau y analizar las implicaciones de esta para el cuidado de sí misma. Se trata de un estudio cualitativoexploratorio con el uso de la Teoría de las representaciones sociales. La colecta de dados fue realizada en el periodo de enero a diciembre de 2007, con el empleo de dos técnicas: la entrevista semi-estructurada con preguntas abiertas y la observación libre. Para la interpretación de esos dados fue utilizada la técnica de análisis de contenido temático. La investigación tuvo como resultado tres unidades temáticas, así denominadas: El examen Papanicolau - un cuidado con la salud de la mujer; Tabúes y creencias sobre el examen Papanicolau; y el Una práctica de cuidado de sí misma: el examen Papanicolau. En el estudio observamos que las mujeres temen mucho tener cáncer cérvico-uterino y, por ese motivo, representan el examen Papanicolau como una práctica de cuidado de sí misma.

Palabras-claves: Enfermería. Neoplasias Uterinas. Frotis Vaginal. Salud de la Mujer.

'Professor Assistente da Faculdade de Enfermagem da Universidade Federal do Pará. Doutorando do DINTER/UFPa/UFSC/CAPES. Mestre em Enfermagem pela EEAN/UFRJ. Membro do Grupo de Estudos de História do Conhecimento de Enfermagem (GEHCE) e do Grupo de Pesquisa: Educação, Políticas e Tecnologia em Enfermagem da Amazônia

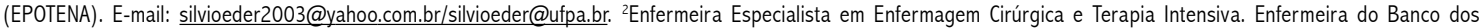
Olhos do Hospital Ophir Loyola de Belém-PA. Membro do Grupo de Pesquisa EPOTENA. ${ }^{3}$ Professora Adjunta da Faculdade de Enfermagem da UFPA. Doutora e Mestra em Enfermagem Fundamental pela EERP/USP. Coordenadora Operacional Local do DINTER/UFPa/UFSC/CAPES. Membro do Grupo de Pesquisa EPOTENA. ${ }^{4}$ Professora Assistente da Faculdade de Enfermagem da UFPa. Doutoranda do DINTER/UFPa/UFSC/CAPES. Mestra em Saúde Pública pela FIOCRUZ. Membro Grupo de Pesquisa Cuidando e Confortando $(C \& C)$ e do Grupo EPOTENA. ${ }^{5}$ Enfermeira graduada pela Faculdade de Enfermagem da UFPA. ${ }^{6}$ Enfermeira graduada pela Faculdade de Enferma 


\section{CONSIDERAÇÕES INICIAIS}

Esta pesquisa tem como objeto de estudo as representações sociais de mulheres amazônidas sobre o exame Papanicolau e suas implicações para o cuidado de si mesmas. 0 câncer do colo do útero é o segundo mais freqüente entre as mulheres no mundo e é culpado, anualmente, por cerca de 471 mil casos novos e pelo óbito de 230 mil mulheres por ano. A incidência deste tipo de câncer torna-se mais evidente na faixa etária de 20 a 29 anos, sendo que o risco aumenta rapidamente até atingir seu ápice na faixa etária de 45 a 49 anos. Cerca 80\% dos casos novos ocorrem em países em desenvolvimento, onde, em algumas regiões, é o câncer mais comum entre as mulheres. Ressaltamos que a sobrevida média estimada em países desenvolvidos é de $59 \%$ a $69 \%$. Já nos países em desenvolvimento, os casos são detectados em estágios mais avançados da doença, propiciando uma sobrevida média de $49 \%$ após cinco anos ${ }^{1}$.

No Brasil, o número de novos casos de câncer no colo do útero esperados em 2008 será de 18.680, com o risco estimado em 19 casos a cada 100 mil mulheres. Este tipo de câncer, sem levarmos em consideração os tumores de pele não melanoma, é o mais incidente na Região Norte (22/100.000), sendo que no Estado do Pará a incidência será de 790 casos novos; destes, 400 se concentrarão na capital'.

Durante a realização da VI Conferência Mundial sobre a Mulher, realizada na China, em setembro de 1995, o Governo Federal, por meio do Instituto Nacional do Câncer (INCA), reconheceu a necessidade da implantação de um programa de âmbito nacional priorizando o controle do câncer de colo de útero no Brasil. Um grupo de técnicos do Ministério da Saúde, em parceria com distintos organismos nacionais e internacionais, preparou um estudo piloto que, posteriormente subsidiaria o Programa Nacional de Controle do Câncer do Colo do Útero (PNCCCU), que foi denominado Programa Viva Mulher².

0 Projeto Piloto Viva Mulher foi implantado a partir do primeiro semestre de 1997 em cinco capitais brasileiras: Curitiba, Brasília, Recife, Rio de Janeiro e Belém e no Estado de Sergipe. A experiência acrescentada por meio do Projeto Piloto Viva Mulher, no período de 18 de agosto a 30 de setembro de 1998, favoreceu a extensão nacional, denominada Fase de Intensificação. Nesse período foram empregadas táticas para estruturação da rede assistencial e estabelecimento de um sistema de dados para o monitoramento das ações e dos mecanismos para mobilização e apreensão de mulheres para controle. A partir de 1999 foi principiada a Fase de Consolidação, baseada na análise e reestruturação das estratégias empregadas nas duas etapas anteriores, com a inserção das ações de controle do câncer de mama no Programa².

O programa Viva Mulher no Estado do Pará emergiu como um normatizador da realização do exame Papanicolau, pois adotou kits para coletas, padronizou os procedimentos de coleta e a apontou a importância da resolutividade do observar e tratar com ênfase na educação das usuárias. 0 referido programa teve aspectos positivos como: a desburocratização do exame, 0 estabelecimento do fluxo de atendimento do nível de atenção primária a terciária, o consenso para periodicidade de realização do exame, o aumento das unidades cadastradas para coleta ao final da municipalização e a parceria com a sociedade civil ${ }^{3}$.

0 programa Viva Mulher também teve seus contrapontos; 0 maior problema foi o de aspecto político, já que se encontrava uma rede com poucos recursos e dividida entre estados e municípios, além da ausência de unidades de saúde em alguns municípios. Outros problemas que destacamos foram de recursos humanos para a coleta, o preparo e a leitura das lâminas, e de funcionários desmotivados para atender as metas das Unidades Básicas de Saúde (UBS) do Estado do Pará. A estrutura física inadequada para realização do serviço também colaborou como um agravante para a implementação do programa ${ }^{3}$. A partir de sua implantação, o referido programa propiciou a inserção do exame do Papanicolau no cotidiano da mulher amazônida

Outro ponto que evidenciamos é que o principal agente etiológico do câncer de colo de útero é o vírus do papiloma humano (HPV), que é transmitido por via sexual. Aproximadamente todos os casos de câncer de colo uterino são ocasionados por um dos 15 tipos oncogênicos do HPV, sendo os mais comuns o HPV 16 e o HPV 18, esse último predominante no Pará. Outros fatores que contribuem para a etiologia da doença são: o tabagismo, o uso de anticoncepcionais orais, baixa ingesta de vitaminas, multiplicidade de parceiros sexuais e iniciação sexual precoce ${ }^{1}$.

0 exame Papanicolau, até a década de 90 , foi a principal estratégia empregada em programas de rastreamento do câncer do colo de útero. Atualmente existem novos métodos, como testes de deteç̧ão do DNA do HPV e a inspeção do colo do útero com o ácido acético (VIA) ou lugol (VILI). Porém, destacamos que no Brasil o exame citopatológico é a estratégia de rastreamento preconizado pelo Ministério da Saúde, devendo ser priorizado para mulheres na faixa etária de 25 a 59 anos'.

Ressaltamos que é estimado que uma redução de cerca de $80 \%$ da mortalidade por este tipo de câncer pode ser alcançada por meio do rastreamento de mulheres na faixa etária de 25 a 65 anos com o teste Papanicolau, que propicia a deteç̧ão precoce de lesões precursoras com alto potencial de malignidade ou carcinoma in situ, sendo necessário garantir a organização, a integralidade e a qualidade do programa de rastreamento, assim como a busca ativa de pacientes. A agência de regulamentação americana, a Food and Drug Administration (FDA), e a Agência de Vigilância Sanitária (ANVISA/MS), seguindo o exemplo de vários países, aprovaram a comercialização da primeira vacina para prevenção das infecções que ocasionam o câncer do colo do útero (HPV 16 e 
18). 0 emprego da referida vacina pode se constituir, futuramente, em uma ferramenta relevante para o controle deste tipo de câncer ${ }^{1}$.

Enfatizamos que o Pará está localizado na Amazônia Legal, que corresponde a 4,8 milhões de $\mathrm{Km}_{2}$, isto é, ocupa mais da metade do território nacional. Este aspecto é relevante, pois propicia que a população do referido estado se identifique como amazônida. Esse é compreendido como o indivíduo que vive em profunda relação de harmonia com a natureza que o cerca, 0 que gera fecunda produção de imaginário próprio que se instaura como uma zona indistinta entre o real e o surreal, onde os rios e as florestas tornam-se muito importantes para esse cotidiano ${ }^{4}$.

A mulher amazônida é detentora de um conjunto de crenças, que se não forem bem trabalhadas podem contribuir para sua não-adesão a uma determinada prática de saúde, no caso deste estudo a realização do exame Papanicolau. Cabe enfatizarmos que os valores culturais sem correlação com a realidade podem representar um grande obstáculo para os profissionais que atuam na promoção da saúde e na prevenção de doenças ${ }^{5}$.

A realização dos programas de prevenção do câncer de colo uterino na educação pública torna-se exemplo de um esforço organizado para realizar diagnósticos precoces, por meio de uma técnica de educação em massa. Porém, esse processo educacional tem que respeitar o acervo cognitivo do qual a mulher amazônida é detentora, para que possa atingir seu objetivo - o controle deste tipo de câncer. Por tal motivo percebemos a relevância de se desvelar suas representações sociais sobre o exame Papanicolau, pois investigar esse objeto psicossocial é relevante para se compreenderem os aspectos subjetivos que influenciam nas condutas e atitudes das mulheres amazônidas no cuidar de si mesmas.

A partir do exposto, delimitamos os seguintes objetivos: descrever as representações sociais de mulheres amazônidas sobre o exame Papanicolau e analisar as implicações dessas representações para o cuidado si mesma.

\section{METODOLOGIA}

Esta pesquisa é do tipo exploratório-descritivo, com o emprego de uma abordagem qualitativa, adotando como suporte teórico-conceitual as representações sociais criadas por Serge Moscovici, que as define como uma forma de conhecimento que possui uma maneira especíica de compreender e comunicar o que nós já sabemos, tendo como objetivo abstrair sentido do mundo e introduzir no mesmo ordem e percepções, que reproduzam o mundo de uma forma significativa ${ }^{6}$. A referida teoria trabalha com o cognitivo, sua interação com o meio social, atuando como um agente transformador do cotidiano. Essa teoria evidencia como um grupo re-presenta e constrói uma realidade ${ }^{7}$.
Os sujeitos foram 20 mulheres que freqüentam a Unidade Municipal de Saúde no bairro do Telégrafo, localizada no Município de Belém, para realizar o exame preventivo. 0 estudo foi baseado nos seguintes critérios: as mesmas deveriam estar orientadas no tempo e no espaço, estarem cientes quanto à finalidade da pesquisa e consentirem seus depoimentos, por meio da assinatura do Termo de Consentimento Livre e Esclarecido.

Quanto ao aspecto ético, o projeto da pesquisa foi submetido ao Comitê de Ética e Pesquisa da Universidade do Estado do Pará (UEPA), localizado no Campus IV do Centro de Ciências Biológicas e da Saúde, tendo sido aprovado na reunião do referido Comitê de 30/06/2006. 0 mesmo atendeu à Resolução n 196/96 do Conselho Nacional da Saúde. Para preservar o anonimato das depoentes, empregamos o sistema alfanumérico para identificação dos seus relatos.

A coleta de dados foi realizada no período de janeiro a dezembro de 2007, utilizando-se duas técnicas de coleta: a entrevista semi-estruturada com perguntas abertas e a observação livre, aplicadas na mesma seqüência. Foi utilizado um questionário para identificação do perfil sócio-cultural dos sujeitos do estudo, constando de: idade, cor/raça, naturalidade, estado civil, início da atividade sexual, paridade, tabagismo, grau de instrução, renda, seguridade social para saúde, religião, condições habitacionais e saneamento básico.

A entrevista semi-estruturada foi composta por questões abertas pelo fato de a mesma permitir o direcionamento pelo entrevistador, de forma flexível, podendo ser assumido pelo entrevistado. Por fim, empregamos a observação livre que foi usada como uma técnica complementar, pois acreditamos que ela favorece a compreensão da linguagem não-verbal, ou seja, comportamentos, expressões faciais e físicas presentes durante a entrevista dos sujeitos de estudo, auxiliando-nos na análise dos dados e na conclusão do estudo.

Para procedermos à análise e interpretação dos dados, optamos em utilizar a técnica de análise temática por sabermos que esta favorece o descobrimento dos núcleos de sentidos que compõem a comunicação e a freqüência, contribuindo para melhor compreensão do texto do discurso ${ }^{8}$. A autora desdobra esta modalidade de análise em: $1^{\circ}$ pré-análise - nesta etapa organizamos os depoimentos, após a realização da leitura flutuante e a constituição do corpus; a $2^{\circ}$ etapa foi a de exploração do material; posteriormente realizamos a $3^{\circ}$ etapa, que consistiu no tratamento dos dados. A partir das referidas etapas emergiram três unidades temáticas: "O Papanicolau um cuidado com a saúde da mulher"; "Tabus e crenças sobre o exame Papanicolau"; e "Uma prática de cuidado de si mesma: o exame Papanicolau". 


\section{REPRESENTAÇÕES SOCIAIS DO EXAME PAPANICOLAU PARA AS MULHERES AMAZÔNIDAS}

\section{O Papanicolau - um cuidado com a saúde da mulher}

0 exame Papanicolau é de grande utilidade para a prevenção do câncer cérvico-uterino, sendo sua realização extremamente importante para diagnóstico precoce deste tipo de câncer. Destacamos que o exame faz parte do universo dessas mulheres, ainda que por diferentes motivos; logo, se transforma em um objeto psicossocial, pois este conhecimento novo é incorporado pelo indivíduo que o compartilha com seu grupo de pertença.

Nesta unidade percebemos que, do total de depoentes, dez $(50 \%)$ evidenciaram a relação de se fazer o exame Papanicolau como um ato de cuidado a saúde. Desta maneira, ao se prevenir a doença, esta não seguiria seu percurso, e não se tornaria um problema de saúde para as mulheres, como nos mostra o seguinte relato:

[...] o exame preventivo é muito importante, porque ele vai detectar não só o começo do câncer, mas as doenças em si, DST, condiloma e outras bactérias também, os fungos, gardnerella, NIC I, NIC II, NIC III; muito importante fazer o preventivo [...] (E3).

Destacamos que o saber reificado sobre as finalidades do exame Papanicolau, presente no depoimento acima, serviu como matéria-prima para construção de uma representação social que atribuiu a ele um significado relevante para saúde da mulher. Este contexto contribuiu para elaboração de um comportamento prático - o cuidado de si pautado na atitude de se submeter ao exame preventivo. Este momento pode ser observado nos depoimentos a seguir:

[...] sempre eu ensino pras minhas filhas também: vão que isso aí é muito bom a gente fazer, a gente tem que se cuidar[...] (E4).

É fundamental que os serviços de saúde orientem a população sobre o que é, e qual a importância do teste de Papanicolau, pois esse saber erudito favoreceu, no estudo, 0 surgimento do conhecimento consensual que atrelou o exame a uma prática de saúde. Destacamos que, apesar do câncer do colo uterino poder ser prevenido, ainda há mulheres que desenvolvem este tipo de câncer e morrem no Brasil, pelo fato de desconhecerem a finalidade do exame.

Constatamos, durante as entrevistas, que nove (45\%) depoentes sabiam da importância da realização do referido exame como uma forma de diagnosticar precocemente não só o câncer cérvico-uterino, mas também outras doenças.
[...] É uma coisa muito importante pra nós mulheres, porque é uma questão de se prevenir, uma questão até de viver um pouco mais, de ver como é que você está mesmo por dentro. 0 correto mesmo é você fazer o seu exame ano a ano pra poder se prevenir de tantas coisas, porque através do exame preventivo, você não descobre só um câncer, mas outras doenças [...] (E1).

0 programa de prevenção do câncer cérvico-uterino, juntamente com o programa de prevenção do câncer de mama, foi criado como uma forma de cuidar da saúde da mulher, visto que as estatísticas apontam um crescimento acentuado de mulheres acometidas pelo câncer. Por este motivo, surgiu a preocupação por parte do Governo, junto ao Ministério da Saúde, em criar os referidos programas implantados na rede básica de saúde, onde as mulheres têm acesso às informações a respeito das formas preventivas, diagnóstico e tratamento. Um dos objetivos principais do programa de prevenção do câncer cérvico-uterino é a detecção precoce do câncer para evitar que a doença se instale, atingindo estágios mais avançados, dificultando, assim, o tratamento, já que o sucesso do tratamento depende de um diagnóstico precoce ${ }^{9}$.

É fato que o câncer é uma doença crônico-degenerativa e que, no caso do câncer cérvico-uterino, tem uma evolução lenta, favorecendo, assim, a utilização de métodos preventivos como uma forma de permitir um diagnóstico precoce e uma terapêutica eficiente ${ }^{10}$. Constatamos que dez (50\%) depoentes sabiam da importância da realização do exame para a prevenção dos estágios mais avançados da doença, como podemos observar nos seguintes relatos:

[...] pra mim é uma maneira de nós, mulheres, prevenirmos o câncer, porque o câncer pode ser prevenido logo no início. Eu entendo assim $[\ldots](E 20)$.

0 exame Papanicolau é um método de prevenção barato e acessivel, e qualquer mulher tem o direito legal de ir a uma unidade de saúde e solicitá-lo. Porém, sabemos que, pelo fato de o Pará ter uma grande extensão territorial, surgem dificuldades no acesso aos serviços de saúde e, por conseguinte, ao exame. Esta realidade é vivenciada por mulheres que residem em localidades mais distantes da capital.

0 sistema de saúde muitas vezes considera a mulher importante apenas no que diz respeito à função reprodutora. Ultimamente, o problema tem se agravado com a questão do planejamento familiar, que tem como objetivo, de forma prioritária, o controle da população, deixando a saúde, o bemestar e o direito da mulher a um plano muito secundário. Isto é refletido, de forma bem clara, na própria organização do sistema 
e nos índices de mortalidade e morbidade de mulheres em nosso meio ${ }^{10}$.

As mulheres ainda têm no câncer do colo uterino uma das principais causas de morte por neoplasia. Como seres que pensam e têm uma cultura própria, precisam ser vistas integralmente e não apenas pelo sintoma mais evidente; mas também procurar encontrar respostas às suas angústias, reconhecer as suas emoções, pode ser tanto ou mais importante para seu o conforto e sua saúde que o mais preciso dos diagnósticos biomédicos ${ }^{10}$.

0 que observamos no estudo é que as depoentes já se preocupam mais com sua saúde, seja por medo de ter o câncer, pela influência da mídia ou pelo do próprio grupo que ela faz parte, e esse processo é evidenciado nas suas representações sociais. Pelo fato de esta ser uma maneira de interpretar e de pensar a realidade que nos cerca, é uma forma de conhecimento social ${ }^{11}$.

As representações sociais intervêm de várias maneiras: pelo contexto concreto onde estão situados pessoas e grupos; pela comunicação que estabelece entre eles; pelos quadros de apreensão fornecidos por sua bagagem cultural; pelos códigos, valores e ideologias ligados às posiç̃es ou participações sociais específicas $^{10}$. Esse compartilhamento de informações e experiência entre o grupo foi observado na fala de seis (30\%) depoentes:

\section{[...] tem muita gente que mora perto do posto de saúde, muita gente não tem interesse em se cuidar, quando vai perceber já é tarde demais. $A$ gente vê tanto caso por aí, eu fico preocupada, eu converso muito com outras mulheres, penso que quanto mais cedo procurar o exame émelhor [...] (E10).}

Essa troca de informação entre as mulheres da pesquisa repercute de maneira positiva para a prevenção deste tipo de câncer; portanto, todos os profissionais devem estar atentos a esta questão e praticar de forma mais ativa a prevenção e promoção da saúde, provocando, assim, mudanças de caráter prático que estimulem as mulheres a dar mais atenção a sua saúde, e realizar seus exames periódicos com a finalidade de evitar a morbidade e mortalidade de muitas delas pelo câncer, uma doença que, se tratada logo no início, tem maiores chances de cura.

Apesar de constatarmos que muitas depoentes sabem da importância do exame Papanicolau, pois é representado como um exame de quem se cuida, a Enfermagem precisa continuar investindo no cuidado preventivo, como forma de ampliar cada vez mais o número de mulheres que cuidam de sua saúde. Destacamos como estratégia que pode ser adotada pela Enfermagem a orientação em todas as ocasiões surgidas, seja em uma palestra, uma consulta de rotina ou uma visita domiciliar. Esse compromisso deve ser assumido não só pela Enfermagem, mas por todos os profissionais da área da saúde.

\section{Tabus e crenças sobre o exame Papanicolau}

Nesta unidade constatamos a existência de fatores que contribuíam para recusa das depoentes em se submeter ao exame Papanicolau, tais como: a desinformação, a falta do costume de se prevenir da doença, a dificuldade de acesso às unidades municipais de saúde e a proibição de alguns maridos. Porém o tabu mais freqüente entre as depoentes surge com a dificuldade na hora da realização do exame, pelo fato de algumas mulheres ainda terem receio de sentir dor, medo ou vergonha.

\section{[...] É constrangedor, mas é uma coisa que a gente tem que fazer. Eu acho que deve ser constrangedor pra mulher isso. Porque eu acho que a gente, mulher, a gente tem um pouco de vergonha [...] (E5).}

Uma representação social se forma no cotidiano do indivíduo, por meio da captação das idéias que circulam no seu grupo social ${ }^{6}$. Percebemos que muitos comportamentos em algumas depoentes foram influenciados pelo grupo social ao qual pertenciam no que diz respeito à questão da vergonha $\mathrm{e}$ do medo de realizar o exame, ou seja, elas já foram com uma idéia prévia de que seria um procedimento que causaria dor e constrangimento. Esta realidade se fez presente devido à priorização do ato de se fazer o exame Papanicolau, ficando a informação da usuária sobre a finalidade do mesmo em segundo plano.

Os enfermeiros têm um papel fundamental na questão da prevenção, devendo exercer um papel de educador, trabalhando mais na questão do conhecimento das representações sociais que influenciam as mulheres no seu cuidado de si. Desta maneira, deixa-se de lado o tradicional modelo biomédico, 0 qual só se preocupa em tratar doenças, e incentivam-se as mulheres a adotarem práticas preventivas, não permitindo, assim, o crescimento das estatísticas de morte por câncer cérvico-uterino, doença esta que pode ser tratada, desde que seja diagnosticada precocemente, por meio de um simples exame-o Papanicolau.

Percebemos também a importância da divulgação, por meio da mídia, da necessidade da realização do exame Papanicolau, segundo preconiza o Ministério da Saúde, pois sabemos que os meios de comunicação em massa exercem uma forte influência no comportamento das pessoas, tanto para beneficiar quanto para prejudicar a saúde.

A mídia, nesse caso, deve ser usada de maneira positiva, tentando divulgar o benefício que o preventivo traz para a saúde da mulher, pois sabemos que ela tem um grande poder na formação de representações sociais. Ressaltamos a necessidade de desmistificar o exame Papanicolau, eliminando a vergonha e o medo que muitas mulheres ainda têm ao procurar o serviço de saúde.

Essa atitude é uma questão cultural, pois vivemos em uma sociedade patriarcal em que a mulher durante muito tempo foi reprimida pela figura masculina, primeiro pela dominação do 
pai, depois pela do marido, que muitas vezes não incentiva sua esposa, sua irmã e sua mãe a realizarem seus exames, externando assim uma atitude de domínio.

Como já foi dito anteriormente, ainda existe vergonha, preconceito e falta de informação com relação ao assunto em questão. Com o devido esclarecimento prestado às mulheres, a tendência é que as mesmas vejam a realização do exame Papanicolau como uma prática rotineira e freqüente em suas vidas, evitando que o serviço de saúde seja procurado somente quando já apresentam algum sintoma de doença.

Um aspecto positivo que observamos foi que, apesar da vergonha estar presente no cotidiano de muitas mulheres entrevistadas, nove $(45 \%)$ delas referiram que, embora assumam a vergonha que sentem de realizar o exame Papanicolau, são conscientes de que é importante fazê-lo deixando o constrangimento e a timidez em segundo plano:

[...] Acho que a gente tem que deixar de lado a vergonha e fazer mesmo porque a nossa saúde é mais importante [...] (E12).

Ressaltamos que, para que a realidade evidenciada aqui seja superada no cotidiano da mulher amazônida, é necessário um conjunto de ações educativas desenvolvidas com a participação da comunidade, no sentido de ampliar o conhecimento sobre os fatores de risco, o desenvolvimento da doença e a importância da realização periódica do exame Papanicolau.

Vimos, no decorrer das entrevistas, que algumas de nossas depoentes sabem da importância de campanhas educativas por parte dos profissionais de saúde, como se segue:

\section{[...]Eu acho que mais campanhas deveriam haver, não só pra cá, mas pra outros interiores, pra poderem nos orientar melhor, porque ainda tem mulheres na cidade que ainda têm aquela vergonha. Porque não se deve ter vergonha de fazer uma prevenção, de se cuidar[...] (E1).}

Reconhecemos que, apesar de ser necessária a educação por parte dos profissionais de saúde, muitas mulheres não apresentam motivação individual para encarar o câncer como uma realidade que, a qualquer momento, pode surgir em suas vidas. Neste ponto, o cuidado deficiente de si mesma pode acabar interferindo, de forma prejudicial, na prevenção e detecção precoce da doença. Esta realidade emergiu nas falas de cinco $(25 \%)$ das depoentes:

[...] A minha mãe já viu muitas pessoas, mulheres novas da idade dela morrendo porque não se preveniram, não tiveram essa força como nós tivemos de ir e fazer o exame antes que a doença ficasse mais séria [...] (E20).
A mulher durante muito tempo teve vergonha de expor seu corpo; essa questão vem mudando ao longo dos anos, porém ainda hoje encontramos mulheres que ainda sentem constrangimento de ir ao serviço de saúde por temerem serem examinadas. Sabemos que o exame Papanicolau expõe o corpo da mulher, que, para muitas delas, é sagrado, como foi observado durante nossa pesquisa.

Felizmente, em nossa pesquisa, a maioria das mulheres relata que o exame Papanicolau é muito importante para a saúde delas. Outras reconhecem que, apesar de ser um exame constrangedor, têm a consciência de que precisam realizar, porém reconhecem que muitas mulheres não realizam por ainda terem vergonha de realizar.

Ficou evidente nesta unidade que, apesar de tudo que se tem feito até hoje em nível de prevenção sobre o câncer do colo uterino, ainda não podemos considerar suficiente porque ainda temos o surgimento de muitos casos novos. Além de incentivarmos as campanhas sobre a realização do exame Papanicolau, precisamos também conhecer e valorizar as representações sociais das mulheres sobre o exame, para que possamos compreender seus medos e suas angústias, procurando um atendimento de forma integral à saúde da mulher.

\section{Uma prática de cuidado de si mesma: o exame Papanicolau}

0 exame Papanicolau, por ser um exame capaz de detectar uma doença muito grave, como o câncer de colo de útero, foi representado por dez (50\%) das depoentes como um ato de cuidado de si mesma, conforme destacado no relato a seguir:

\section{[...] O exame preventivo é uma responsabilidade comigo mesma, um cuidado comigo mesma, preocupação, porque hoje em dia as mulheres não se preocupam consigo mesmas [...] (E16).}

Destacamos que o fato de muitas mulheres representarem o câncer do colo do útero como uma doença distante delas favorece que não realizem o exame Papanicolau, e, na maioria das vezes, esse conhecimento consensual só é mudado quando elas são acometidas pela doença ou o é alguém conhecido.

Outro aspecto que destacamos foi que metade das mulheres entrevistadas expressou preocupação com sua saúde e com seu bem-estar físico, englobando aspectos tanto físicos quanto psicológicos. A mulher deve ser concebida como um ser integral, biológico e psicossocial, parece um conceito simples; 0 costumeiro, porém, é analisar apenas o órgão doente, e não a mulher inteira como um ser que sente, pensa e que deve ser vista integralmente, e não apenas pelo sintoma mais evidente ${ }^{10}$. A preocupação com a saúde entre as depoentes pode ser observada nos relatos a seguir:

[...] Eu cuido da minha saúde, eu quero saber da minha saúde, [...] A nossa saúde é preciosa, é importantíssimo [...] (E11) 
Atualmente o sistema volta-se apenas para pacientes que se julgam com sintomas capazes de justificar a consulta a um profissional de saúde. A essa situação, o governo tem respondido com a construção de mais hospitais, quando se deveria investir na atenção primária. Isto faz com que 0 atendimento de emergência represente hoje cerca de metade das consultas médicas oferecidas pelo sistema de saúde ${ }^{10}$. Reforçamos que 0 atendimento primário deve cobrir pelo menos $65 \%$ do total de consultas, ficando $20 \%$ para o atendimento especializado e apenas $15 \%$ para 0 atendimento de emergência ${ }^{12}$.

Na verdade, não haveria necessidade de um único leito no país se o governo estivesse disposto a investir no atendimento primário, dando-lhe a condição de porta de entrada do sistema. Este fato prejudica a procura das mulheres pela prevenção, embora, dentro de nossa pesquisa, algumas relatem terem cuidado com sua saúde e se preocuparem com seu bem-estar físico, como podemos observar:

\section{[...] Às vezes eu fico preocupada: não, vou fazer o preventivo porque é importante pra mim e praticamente pra minha saúde [...] (E18)}

0 câncer como um problema de saúde pública no Brasil é merecedor de grande atenção por parte dos profissionais de saúde, em especial da Enfermagem, que pode contribuir, de forma significativa, para o controle da doença, por meio das ações de promoção de saúde, prevenção e deteç̧ão precoce. As ações educativas, quando desenvolvidas com a participação da comunidade, visam ampliar o conhecimento sobre os fatores de risco, o desenvolvimento da doença e sobre a importância da realização periódica do exame Papanicolau.

As mulheres devem ser incentivadas a ter cuidado com sua saúde, não só relacionado ao câncer do colo do útero, mas também a outras doenças, que podem comprometer sua saúde e qualidade de vida. É necessário orientá-las sobre os fatores de risco para o câncer e a sua eliminação, além da importância da prática do cuidado de si. Isso tudo é possível com o emprego de ações educativas, que possam melhorar a qualidade de vida dessas mulheres, para que se sintam úteis à sociedade e conquistem cada vez mais seu espaço, pois sem saúde isso não é possível.

\section{CONSIDERACÕES FINAIS}

Neste estudo evidenciamos as representações sociais de mulheres amazônidas sobre o exame Papanicolau e suas implicações para o cuidado de si mesmas. Ao realizá-lo, pudemos conhecer o seu pensar, sentir e agir em relação à prática de se submeter ao exame, ficando evidente sua efetivação como uma forma de prevenir o câncer do colo do útero.

Ressalvamos que as mulheres amazônidas compartilhavam informações com o seu grupo de pertença, principalmente no que diz respeito à gravidade do câncer de colo de útero, visto que muitas delas presenciaram a doença de perto, seja por adoecimento de um parente próximo ou por um amigo, e isso Ihes causava muito temor.

Verificamos que a maioria das depoentes encara como sendo normal e importante para a saúde o fato de ir a uma unidade de saúde e se submeter ao exame Papanicolau. Porém, muitas delas relataram ter vergonha durante a realização do exame, 0 que é superado pela necessidade de estar fazendo um exame que, segundo elas, vai estar prevenindo uma forma avançada do câncer do colo do útero.

A representação social do exame Papanicolau como uma forma de diagnosticar, não só o câncer cérvico-uterino, mas também outras doenças graves, contribuiu para a preocupação em prevenir o surgimento de tais enfermidades. Observamos que as palavras prevenção e cuidado sempre surgiram nos depoimentos, enfatizando a certeza de que o exame não é apenas um teste de rotina, mas uma prática de cuidado de si mesma.

\section{REFERÊNCIAS}

1. Instituto Nacional do Câncer-INCA. Estimativa 2008: incidência do câncer no Brasil. Rio de Janeiro (RJ); 2007.

2.Instituto Nacional do Câncer - INCA. Viva mulher. Programa Nacional de Controle do Câncer do Colo do Utero. [on-line]. Rio de Janeiro (RJ); 2000 [citado 20 fev 2007] Disponível em: http://www.inca.gov.br/ prevencao/programas/viva_mulher_fasel.pdf

3.Gonçalves APO, Ribeiro JM. Políticas de prevenção do câncer de colo uterino nos anos 90 - Pará. In: Lopes MMB, Polaro SHI, Lima VLA. Realidade amazônica: recortes da enfermagem do Pará. Belém (PA): EDUFPA; 2006.

4.Rodrigues A. Uma estrutura de classificação com enfoque na cultura amazônica. Cienc Inf [on-line] 2005 maio/ago; [citado 20 fev 2007]; 34 (2): [aproxim. 9 telas]. Disponível em: http://www.scielo.br.

5.Carvalho ALS, Nobre RNS, Barros SKS, Bezerra SIS, Leitão NMA, Pinheiro AKB. Sentimentos vivenciados por mulheres submetidas a tratamento para papillomavirus humano. Esc Anna Nery Rev Enferm 2007 jun; 11 (2): 248-53.

6.Moscovici S. Representação social da psicanálise. Rio de Janeiro (RJ): Zahar; 1978.

7.Silva SED, Souza MJ. Alcoolismo: representações sociais de alcoolistas abstêmios. Esc Anna Nery Rev Enferm 2004 dez; 8 (3): 420-27.

8.Bardin L. Análise de conteúdo. Lisboa (PO): Ed 70; 1977.

9.Ministério da Saúde (BR). Relatório técnico. Brasilia (DF); 1999.

10.Faúndes A, Zeferino LC, Pinotti JA. Conceito de atenção integral à saúde da mulher. In: Habbe HW. Tratado de Ginecologia. $2^{\mathrm{a}}$ ed. São Paulo (SP) Roca; 1998. v.1.

11.Jodelet D. As representações sociais. Rio de Janeiro (RJ): Ed UERJ; 2001.

12.Linard AG, Silva FAD, Silva RM. Mulheres submetidas a tratamento para câncer de colo uterino-percepção de como enfrentam a realidade. Rev Bras Cancerol 2002 dez; 48 (4): 493-98. 


\section{NOTA BIBLIOGRÁFICA}

a 0 artigo foi extraído do trabalho intitulado: "Esse tal de Nicolau: representações sociais de mulheres sobre o exame preventivo", que foi apresentado na SESSÃO INTERATIVA DE PÔSTER na V Jornada Internacional e III Conferência Brasileira sobre Representações Sociais, que abordou o tema: "Interdisciplinaridade e diversidade de paradigmas", realizadas em Brasília-DF, de 31 de julho a 03 de agosto de 2007. 